\title{
Linear Control Theory for Modelling, Designing, and Performance Evaluation of ATM Congestion Control Algorithms
}

\author{
Saverio Mascolo \\ Dipartimento di Elettrotecnica ed Elettronica \\ Politecnico di Bari, Via Orabona 4, 70125 Bari, Italy \\ Email: mascolo@poliba.it
}

\begin{abstract}
This paper exploits classical control theory to design congestion control algorithms for "best effort" traffic in ATM networks. The control goal is the full utilisation of network links without incurring cell losses. A fluid model approximation of cell flows is assumed and linear differential equations are used to model the dynamics of network queues in response to ABR traffic and quality constrained $(C B R+V B R)$ traffic. The available $A B R$ bandwidth is modelled by means of an unknown and bounded disturbance function. A general end to end control algorithm that feeds back to the source the space that is free in the network buffers is included in the model and a particular one is proposed which is based on Smith's principle. The linearity and the simplicity of the proposed control law allow us to prove, via mathematical analysis, that the algorithm always guarantees no cell losses whereas full utilisation of network links is ensured if the capacity of per-VC buffers is at least equal to the VC bandwidth-delay product. Moreover, per-VC queuing easily allows switches to enforce fairness. Finally, it is shown how performance evaluation can be easily carried out using SIMULINK for MATLAB, which is a software tool widely used by control engineers to simulate dynamic systems. In this way, the effort to develop discrete event simulations is saved.
\end{abstract}

\section{INTRODUCTION}

In recent years, intense research efforts have been focused on the issue of transmitting multimedia traffic over a fully integrated universal network. To this purpose, Broadband Integrated Service Digital Networks (B-ISDNs) 
have been introduced and the emerging Asynchronous Transfer Mode (ATM) technology has been retained the transfer mode to be used in BISDNs (Varaiya and Walrand, 1996). ATM networks seek to provide the end-to-end transfer of fixed size cells and with specified quality of service. The fixed size of the cells reduces the variance of transmission delay making the networks suitable for integrated traffic consisting of voice, video, and data (ATM Forum, 1996; Jain, 1996).

An increasing amount of research has been devoted to different control issues. These research efforts concern with ensuring that users get their desired quality of service. The ATM Forum Traffic Management Group defines five service classes to support multimedia traffic: 1) the Constant Bit Rate (CBR) class, which is conceived for applications such as telephone, video conferencing, and television; 2) the Variable Bit Rate (VBR) class which allows users to send at a variable rate. This category is subdivided into two categories: Real-Time VBR (RT-VBR), and Non-Real-Time VBR (NRT-VBR). An example of RT-VBR is interactive compressed video or industrial control (you would like a command sent to a robot arm to reach it before the arm crashes into something), while that of NRT-VBR is multimedia email; 3) the Unspecified Bit Rate (UBR) class which is designed for those data applications, such as email, file transfer, etc., that want to use any left-over capacity and are not sensitive to cell loss or delay; 4) the Available Bit Rate (ABR) class which is designed for normal data traffic such as file transfer and email. This class does not require cell transfer delay to be guaranteed. However, the source is required to control its rate in order to take into account the congestion status of the network. In this way the Cell Loss Ratio (=Lost Cells/Transmitted Cells) is minimised, and retransmissions are reduced improving network utilisation. It should be pointed out that UBR does not require service guarantee: the drawback is that cell losses may result in retransmissions, which further increase congestion. The ABR service was defined to overcome this problem. It is the only class that responds to network congestion by means of a feedback control mechanism (ATM Forum, 1996; Jain, 1996).

Congestion control is critical in both ATM and non-ATM networks and it is the most essential aspect of traffic management (Jacobson, 1988). A key issue is the "efficient coexistence" of quality-constrained services (CBR+VBR) and "best effort" services (ABR+UBR). Many efforts have been devoted to design control algorithms for ABR input rates. Many of the proposed algorithms lack of a complete theoretical foundation because they are derived using heuristic approaches. Nowadays, the interest for a theoretic approach based on control theory is ever increasing (Benmohamed and Meerkov, 1993). In fact, due to propagation delay, most algorithms exhibit persistent oscillations and can even be unstable. In (Benmohamed and Meerkov, 1993; 1994) an analytic method for the design of congestion controllers, which ensure good dynamic performance along with fairness in 
bandwidth allocation, has been proposed. However the method requires a complex on-line tuning of control parameters in order to ensure stability and damping of oscillations under different network condition. Moreover, it is difficult to prove the global stability of the scheme due to the complexity of the control strategy. A dual proportional-derivative (PD) controller has been proposed in (Kolarov and Ramamurthy, 1997) to simplify the implementation of this algorithm. In (Rohrs and Berry, 1997), a proportional-integral (PI) controller has been proposed, and in (Fulton and $\mathrm{Li}, 1997)$ an explicit rate algorithm (EPRCA) is illustrated which estimates the background traffic (CBR+VBR) and the effective number of active sources.

Algorithms that cannot be completely evaluated via mathematical analysis need to be tested by means of computer simulations in order to investigate their properties such as stability, fairness and full link utilisation. This validation is partial because is restricted only to the simulated scenarios. Moreover, since ATM networks belong to the class of Discrete Event Systems, simulations always require considerable development effort by part of programmers.

In this paper, a fluid model approximation of cell flows is assumed and a classical control approach is used to model the dynamics of network queues in response to input traffic. The proposed model gives a general framework for modelling, designing and performance evaluation of linear feedback control algorithms. Moreover, Smith's principle is chosen to design an efficient algorithm for throttling ABR input rates in high speed ATM networks. The feedback scheme uses circulating Resource Management (RM) cells in the ratio 1/NRM with data cells, while the intermediate nodes along the VC path stamp the space that is free in the buffers in the RM cells (ATM Forum, 1996). Stability and full link utilisation are shown via mathematical analysis even in the presence of large propagation delay. With respect to other congestion control algorithms, such as, for instance, the one reported in (Charny, Clark and Jain, 1995), our control law does not require the measurement of available bandwidth, a hard task in presence of bursty traffic. Unlike the algorithm proposed in (Izmailov, 1995), where links with constant ABR available bandwidth have been assumed, in this work the "best-effort" ABR bandwidth is modelled by means of a time-varying, unknown and bounded disturbance function. In (Zhao, Li and Sigarto, 1997) an algorithm based on $\mathrm{H}_{2}$ optimal control theory has been proposed, where the ABR rate is only adapted to the low-frequency variation of the underlying VBR traffic. With this approach, stability analysis for the controlled network becomes possible assuming the knowledge of VBR traffic characteristic.

The paper is structured as follows: In Sec. II the model of the system is described; in Sec. III the general framework to design closed loop congestion control algorithms is presented; in Sec. IV Smith's principle is proposed to 
design the control law; in Sec. V performance evaluation is carried out via mathematical analysis; in Sec. VI the discrete time form of the control equation is derived; in Sec. VII computer simulations using SIMULINK for MATLAB (Simulink, 1992) are developed and, finally, the conclusions are outlined.

\section{THE MODEL}

\subsection{The network and traffic model}

The communication network can be considered as a graph (Fig. 1) consisting of:

a) A set $N=\{1, . . \mathrm{n}\}$ of nodes (the switches);

b) A set $L=\{1, \ldots \ell\}$ of communication links, each one characterised by the transmission capacity $c_{i}=1 / t_{i}(\mathrm{cells} / \mathrm{sec})$ and the propagation delay $t_{d i}$.

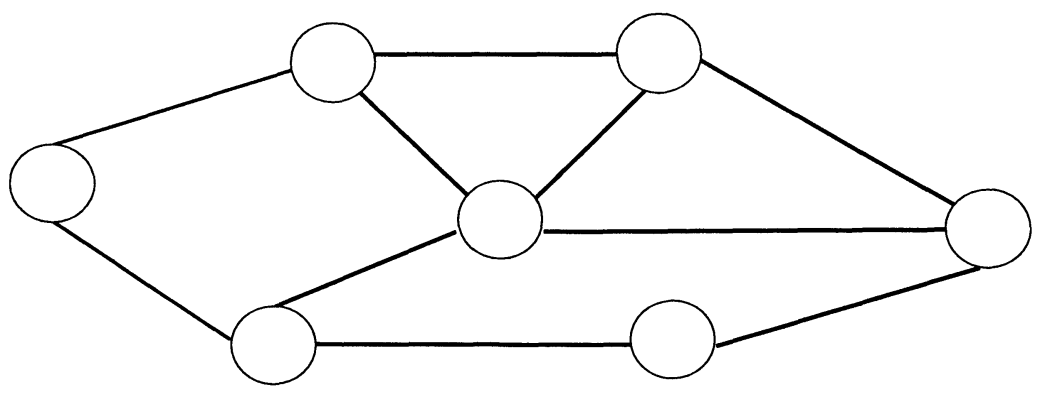

Fig.1: Communication network as a graph of switches and links

Each node is characterised by the processing capacity $1 / t_{p r i}$ (cell/sec) where $t_{p r i}$ is the time the switch $i$ needs to take a packet from the input and place it on the output queue. It is assumed that the processing capacity of each node is larger than the total transmission capacity of its incoming links so that congestion is caused by transmission capacity only. The network traffic is contributed by source/destination pairs $(S, D) \in N \times N$. To each $(S, D)$ connection is associated a Virtual Circuit (VC) mapped on the path $p(S, D)$. Each switch output link maintains a per-VC first in first out (FIFO) queue. A deterministic fluid model approximation of cell flow is assumed, that is, each 
$\mathrm{ABR}$ input rate is described by the function of the time $u(t)$ measured in cells/sec. An ABR source is expected to declare only its peak cell rate, i.e. its maximum transmission speed $c_{s}=1 / t_{s}$. It is worth noting that, in high-speed wide area network, a key parameter is the bandwidth delay product $t_{d j} / t_{j}$, which represents a large number of cells "in flight" on the transmission link.

Remark 1: Per-VC queuing separates cells according to the flow to which they belong. This allows the VCs to be completely uncoupled, that is, each per-VC buffer stores only cells belonging to that VC. The fact that each flow has exclusive buffers allows an algorithm entirely implemented at the source to effectively and easily control congestion. Moreover, the round-robin service can easily enforce fairness (Peterson, L. L. and Davie B. S., 1996).

\subsection{The Feedback Control Scheme}

The closed loop control scheme proposed by the ATM Forum is assumed (ATM Forum, 1996). In this scheme an ABR source sends one control cell (RM cell) every NRM data cells. Each switch encountered by the RM cell along the VC path stamps in the RM cell the space that is free in the buffer associated to the VC only if this value is smaller than the one already stored. At the destination, the RM cell carries the minimum available space over all the encountered buffers and it comes back to the source conveying this value. Upon receiving of this information, the source updates its input rate (Fig. 2).

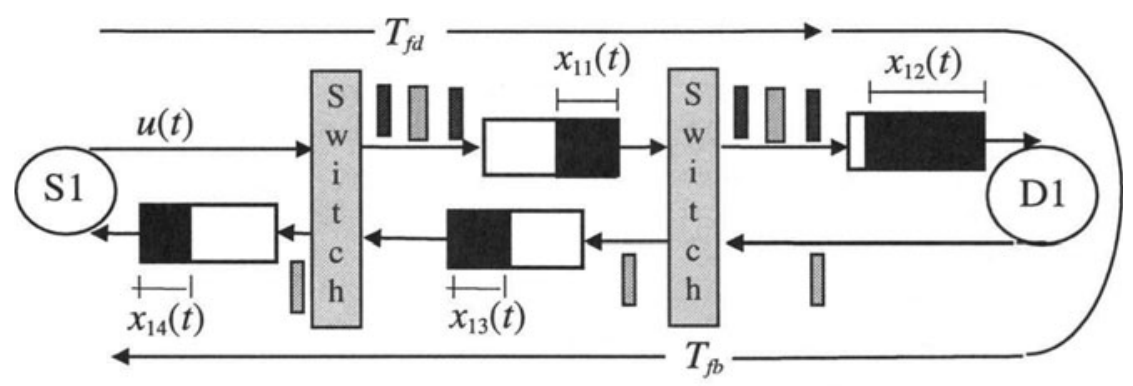

RM cell: Data cell: Congested queue: $x_{12}(t)$

Fig. 2: End to end feedback control scheme using RM cells. 


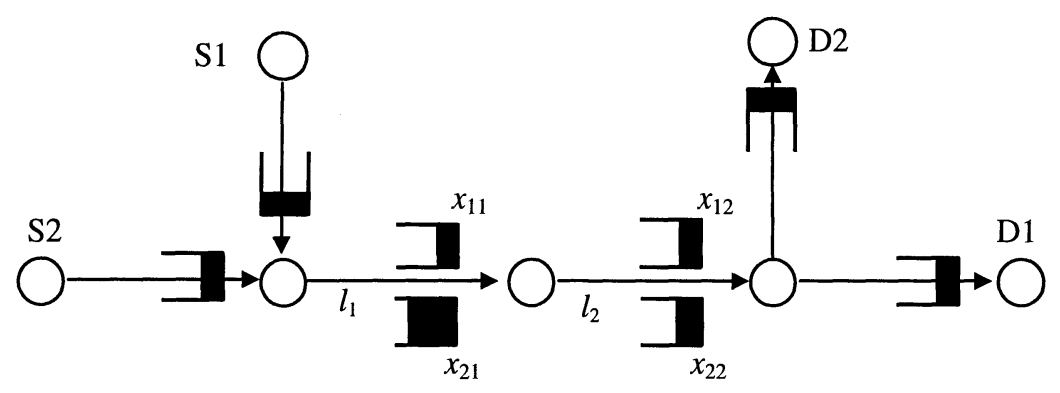

Fig. 3: Two VCs (S1, D1) and (S2, D2) sharing links $l_{1}$ and $l_{2}$ are shown. Per-VC queuing is maintained at output links.

\subsection{The Queue Model}

Two ABR connections, which share links $l_{1}$ and $l_{2}$ and maintain per-VC queuing, are shown in Fig. 3. Let $x_{i j}(t)$ be the level of the queue associated with virtual circuit $\mathrm{VC}_{i}$ and link $l_{j .}$. By writing the flow conservation equation, the queue level at time $t$, starting at $t=0$ with $x_{i j}(0)=0$, is

$$
x_{i j}(t)=\int_{0}^{t}\left[u_{i j}\left(\tau-T_{i j}\right)-d_{j}(\tau)\right] d \tau
$$

where, $u_{i j}(t)$ is the inflow rate due to the $\mathrm{VC}_{i}$ connection, $T_{i j}$ is the propagation delay from the $\mathrm{VC}_{i}$ source to the $x_{i j}$ queue, and $d_{j}(t)$ is the rate of packets leaving the queue, that is, the $A B R$ available bandwidth.

Notice that an output link is shared by ABR, VBR and CBR traffic. Therefore, the buffer depletion rate $d_{j}(t)$ depends on network traffic loading the link. Since it is difficult to measure the available ABR bandwidth, $d_{j}(t)$ is modelled as an unmeasured disturbance.

Fig. 4 shows the block diagram of Eq. (1). Following control systems terminology, the system in Fig. 4 is called the plant. The ABR input rate $u_{i j}(t)$ is the control variable, i.e. the variable that can be throttled. The ABR bandwidth $d_{j}(t)$ is the disturbance, which is assumed to be not measurable. The queue level $x_{i j}(t)$ is the controlled or output variable, i.e. the dynamics that must be reduced to the desired one by means of $u_{i j}(t)$ in the presence of the disturbance $d_{j}(t)$. 


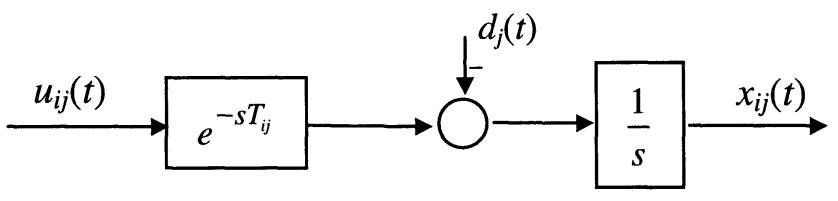

Fig. 4: Block diagram of the plant: $u_{i j}(t)$ is the control variable, $d_{j}(t)$ is the disturbance, $x_{i j}(t)$ is the output variable.

\section{GENERAL FRAMEWORK FOR DESIGNING A LINEAR CONTROLLER FOR ABR TRAFFIC}

In this section, a general framework to design closed loop control algorithms for $A B R$ traffic is proposed. The proposed scheme consists of a closed-loop mechanism feeding back to the ABR source the available buffer space encountered by RM cells along the VC path. The control goals are: 1) stability of network queues; 2) high utilisation of network links; 3) max-min fairness (Jaffe, 1981; Jain, 1996).

\subsection{A linear controller}

The idea of using ABR best-effort traffic to fully utilise bandwidth in ATM networks led to the introduction of closed-loop congestion control algorithms. Binary feedback schemes were first proposed (Jain, 1996; Benmohamed and Meerkov, 1993; Iliadis, 1995; Bonomi, Mitra and Seery, 1995; Yin and Hluchyj, 1994). In these schemes, if the queue level of a switch is greater than a threshold, a binary digit is set in the RM cell. A consequence is that the controlled system is nonlinear even if, in this case, the plant is linear with transfer function: $\exp (-s T) / s$. Moreover, due to the binary feedback conveyed by the RM cells, problems of stability and performance arise.

Following these considerations and noting that RM cells have enough room to store and convey the available buffer space as feedback information, a linear feedback control is designed so that the controlled system (Fig. 5) keeps linear. Linear systems have many appealing properties. Moreover, a complete and well-established control theory exists for them. 


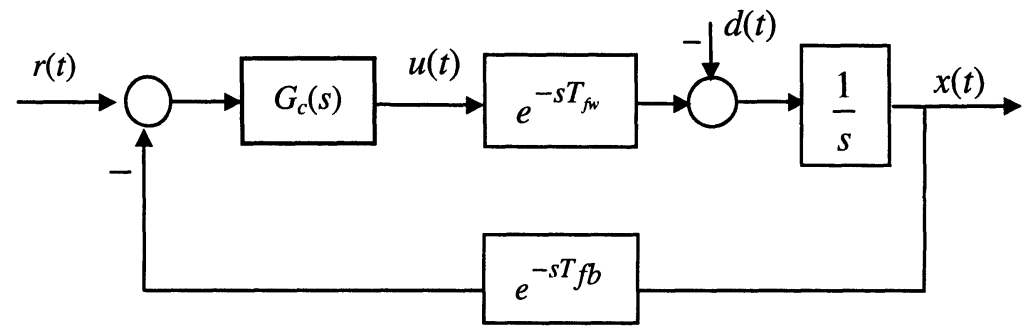

Fig. 5: Block diagram of the controlled bottleneck queue level dynamics $x(t)$ in response to the ABR input rate $u(t)$ and to the available bandwidth $d(t)$.

\subsection{Model of the controlled ABR flows}

Considering a Virtual Circuit, it has a bottleneck link along its path. The buffer feeding this link is the only one with a queue level greater than zero over all the buffers along the path. Thus, the congestion avoidance algorithm must guarantee that: 1) the bottleneck queue does not overflow (i.e. no cell loss); 2) the bottleneck queue level is always greater than zero (i.e. full link utilisation).

The block diagram of the closed loop controlled bottleneck queue dynamics in response to ( $\mathrm{ABR}+\mathrm{VBR}+\mathrm{CBR}$ ) traffic is shown in Fig. 5. In particular, it consists of:

1) The connection bottleneck queue $x(t)^{1}$, which is modelled in the Laplace domain by the integrator $1 / s$;

2) The disturbance $d(t)$, which models the ABR bandwidth that is available for the considered VC; $d(t)$ is an unknown and bounded function that represents the bandwidth left available by coexisting (VBR+CBR+ABR) traffic;

3) The transfer function $e^{-s T_{f w}}$, which models the propagation time $T_{f w}$ from the source to the bottleneck queue;

4) The transfer function $e^{-s T_{f b}}$, which models the propagation time $T_{f b}$ from the bottleneck queue to the destination and then back to the source;

5) The controller transfer function $G_{c}(s)$;

6) The VC input rate $u(t)$;

\footnotetext{
${ }^{1}$ From now on variable subscripts $i j$ are omitted to simplify the notation.
} 
7) The reference signal $r(t)$, which sets a threshold for the bottleneck queue level.

The feedback control scheme works as follows: the source receives the queue level $x\left(t-T_{f b}\right)$ and then inputs the difference $\left(r(t)-x\left(t-T_{f b}\right)\right)$ into the controller $G_{c}(s)$ whose output is the ABR input rate $u(t)$. The input rate reaches the bottleneck queue after the forward propagation delay $T_{f w}$ whereas the buffer free space is fed back to the source after the backward propagation delay $T_{f b}$. We assume that the RM cells have priority over data cells at the queues. As a consequence, the round trip time of the RM cells is constant and equal to the round trip propagation time. A major advantage of this assumption is that random queuing delays are zero and, therefore, the round trip time inside the control loop is reduced to the minimum and is constant.

Remark 2: The round trip time (RTT) of the VC connection is always $T_{f w}+T_{f b}$ wherever the connection queue bottleneck may be positioned along the VC path. Therefore, the proposed scheme also models the realistic case of moving bottleneck.

\section{THE CONTROL LAW}

Fig. 5 shows the general scheme of the proposed linear closed loop control law. The input rate seeks to fill the bottleneck queue, whereas the available bandwidth seeks to empty this queue. To guarantee full utilisation of network links in the presence of the disturbance $d(t)$, the control variable $u(t)$ must pump enough traffic so that the queue is never empty. On the other hand, to guarantee stability, i.e. to avoid congestion, the control $u$ must contain the intensity of data pumping.

Formally, queue stability can be stated as the objective of designing a control law $u(t)$ for each ABR input rate, such that the bottleneck queue level $x(t)$ satisfies the following stability condition:

$$
x(t) \leq r^{o} \text { for } t>0
$$

where $r^{o}$ is the capacity of the buffer. Similarly, full utilisation of network links can be guaranteed if the bottleneck queue level satisfies the following efficiency condition:

$$
x(t)>0 \text { and for } t>T
$$


where $T$ takes into account the round trip propagation time inside the control loop. Clearly, this condition guarantees that any link has always data to send.

Now, setting the reference value $r(t)$ to the bottleneck buffer capacity $r^{o}$, the control action seeks to fill this buffer at its full capacity, whereas the disturbance $d(t)$ tries to empty the queue. Due to the possibly large propagation delays in the control loop, queue level dynamics might exhibit oscillations, and even become unstable. Therefore the design of the linear controller $G_{c}(s)$, which satisfies the stability and efficiency conditions, must be carried out carefully.

We propose to design the controller $G_{c}(s)$ following the Smith principle, which is an important classical control technique for time-delay systems (Smith, 1959; Marshall, 1979; Franklin, Powell and Emami-Naeini, 1994).

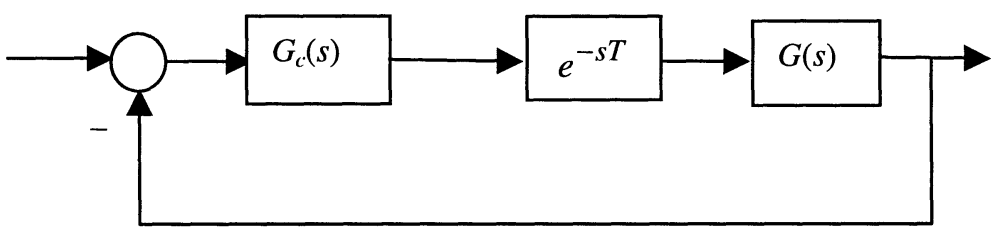

Fig. 6: The linear controller $G_{c}(s)$ and the time-delay system $G(s) \exp (-s T)$

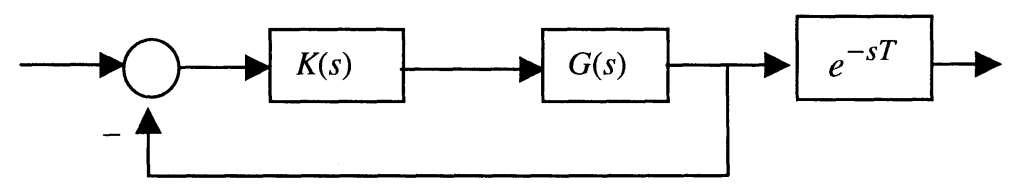

Fig.7: Desired input-output dynamics

\subsection{The Smith principle}

Smith's principle is well-known as an effective dead-time compensator for a stable process with large time delay. Consider the goal of designing a controller $G_{c}(s)$ for the time delay system $G(s) \exp (-s T)$ shown in Fig. 6 . Smith's principle pursues the goal of designing a controller $G_{c}(s)$ such that the resulting closed-loop dynamics is delay-free. More precisely, $G_{c}(s)$ is chosen so that the system becomes equivalent to the reference system 
reported in Fig. 7. This system consists of: 1) the delay-free "plant" $G(s) ; 2)$ the controller $K(s) ; 3)$ the delay $\exp (-s T)$ which is out of the feedback loop.

The chosen reference system is appealing because it is a delay-free system whose output is delayed by the time $T$.

By equating the transfer functions of the systems in Fig. 6 and Fig. 7

$$
\frac{K(s) G(s)}{1+K(s) G(s)} e^{-s T}=\frac{G_{c}(s) G(s) e^{-s T}}{1+G_{c}(s) G(s) e^{-s T}}
$$

the required controller $G_{c}(s)$ results

$$
G_{c}(s)=\frac{K(s)}{1+K(s) G(s)\left(1-e^{-s T}\right)}
$$

The block diagram of the controller is reported in Fig. 8. It should be noted that, using the Smith principle, the problem of designing the controller for the time-delay system in Fig. 6 has reduced to the design of the controller $K(s)$ for the delay-free system in Fig. 7 (Marshall, 1979). Notice that an accurate model of the plant $G(s) \exp (-s T)$ is necessary because this is part of the controller $G_{c}$.

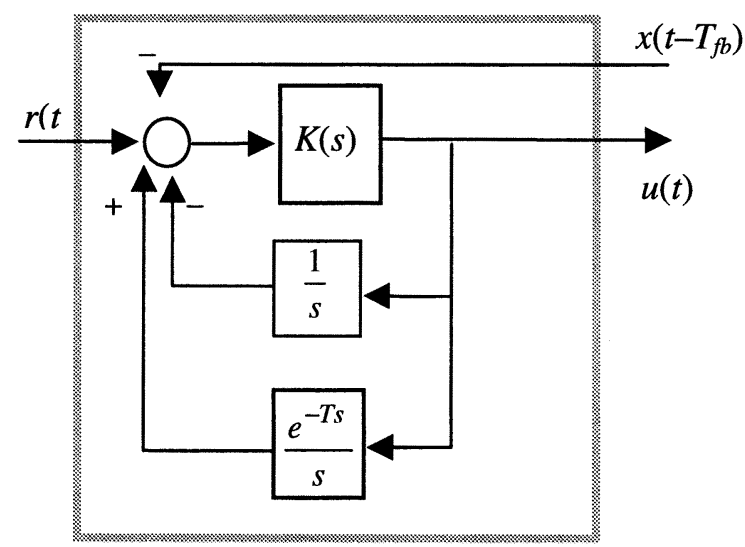

Fig. 8: Block diagram of the controller $G_{c}(s)$ 


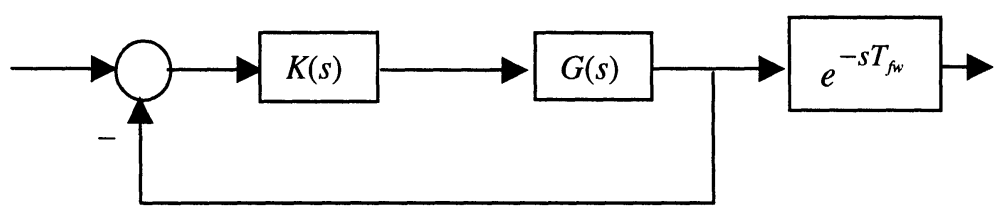

Fig. 9: Desired equivalent system

\subsection{The proposed control law for ABR traffic}

In wide area networks, the round trip time (RTT) of the VC connection strongly influences the stability of the control algorithm. $R T T$ is mostly determined by the propagation delay. Nowadays, ATM vendors are building switches that give priority to RM cells. This assumption reduces the queuing time to zero and makes the round trip time constant and equal only to the round trip propagation time. This quantity is known in advance, when the connection is established ${ }^{2}$. Therefore, since the model of the plant is accurate, Smith's principle can be applied. In particular, the system reported in Fig. 5, where $R T T=T_{f w}+T_{f b}$, can be made equivalent to the delay-free system shown in Fig. 9. In fact, equating the transfer functions in Figs. 5 and 9

$$
\frac{K(s) G(s)}{1+K(s) G(s)} e^{-s T_{f w}}=\frac{G_{c}(s) G(s) e^{-s T_{f w}}}{1+G_{c}(s) G(s) e^{-s\left(T_{f w}+T_{f b}\right)}}
$$

the following controller is derived

$$
G_{c}(s)=\frac{K(s)}{1+K(s) G(s)\left(1-e^{-s \cdot R T T}\right)}
$$

Now, choosing a simple proportional controller $K(s)=k$, where $k$ is a constant gain, and considering that $G(s)=1 / s$, the transfer function of the equivalent system shown in Fig. 9 becomes

$$
\frac{k}{k+s} e^{-s T_{f w}}
$$

\footnotetext{
${ }^{2}$ To take into account the jitter of round trip time due to queuing time, a model containing time varying delays could be considered. However, this would make hard to deal with the system using rigorous and simple mathematical analysis.
} 
This is a first order system with the delay $T_{f w}$ in cascade. From basic control theory it is known that the step response of a first order system delayed by $T_{f w}$ is $r^{0}\left[1-\exp \left(t-T_{f w}\right)\right] \cdot 1\left(t-T_{f w}\right)$, where $r^{0}$ is the magnitude of the step $r(t)=r^{o} \cdot 1(t)$. This response has two advantages: 1) it is bounded by $\left.r^{o} ; 2\right)$ it has no dumped oscillations.

The controller $G_{c}(s)$ is

$$
G_{c}(s)=\frac{k}{1+\frac{k}{s}\left(1-e^{-s \cdot R T T}\right)}
$$

which, in the time domain, gives the following input rate control equation

$$
u(t)=k\left(r^{o}-x\left(t-T_{f b}\right)-\int_{t-R T T}^{t} u(\tau) \cdot d \tau\right)
$$

This is equation is very attractive due to its simplicity. It can be intuitively interpreted as follows: the computed input rate is proportional, through the coefficient $k$, to the available space in the bottleneck buffer, that is $\left(r^{o}-x\left(t-T_{f b}\right)\right)$, decreased by the number of cells released by the VC during the last round trip time $R T T=T_{f w}+T_{f b}=T^{3}$.

We will show that the proposed control scheme guarantees stability of network queues, full utilisation of network links and max-min fairness.

\section{PERFormance EVAluation}

Linear control theory provides an established set of tools which enables us to design algorithms whose performance can be predicted analytically rather than relying on simulations. In particular, to analyse the performance of the proposed algorithm is sufficient to use standard Laplace transform technique. The advantage of mathematical analysis is that it enables properties to be demonstrated in a general setting, whereas the validation using simulations is always restricted to the simulated scenarios. Notice that, in our case, mathematical analysis is possible due to the simplicity of the proposed algorithm and to the assumption of per-VC queuing, which uncouples the flows.

\subsection{The disturbance input}

${ }^{3}$ Each $\mathrm{VC}_{\mathrm{i}}$ is characterized by proper round trip time $T_{i}$ and input rate $u_{i}(t)$. The subscript $i$ is omitted to simplify the notation. 
A link transmission capacity normalised to unity is assumed so that, if all link bandwidth is available for the considered ABR flow, then $d(t)$ is equal to the step function $1(t)$. The coexistence of different ABR sources with VBR and CBR traffic reduces the bottleneck bandwidth that is available for a single VC to $d(t)=1(t)-b(t) \geq 0$, where $b(t)$ is the bandwidth used by the coexisting traffic. By defining $b_{m}=\min _{t}\{b(t)\}$, it results

$$
d(t) \leq 1(t)-b_{m} \cdot 1(t)=a \cdot 1(t)
$$

where $a=\left(1-b_{m}\right) \leq 1$. The step function $a \cdot 1(t)$ is a worst case disturbance that models a bandwidth that is suddenly available at $t=0$.

\subsection{Stability}

Now we show that the controller (4) ensures queue stability in the presence of the disturbance (6).

Proposition 1: The output of the system reported in Fig. 5, where $G_{c}(s)$ is the controller (4), $r(t)$ is the step function $r^{o} \cdot 1\left(t-T_{f b}\right)$ and $d(t)$ is the step function $a \cdot 1(t)$, satisfies the stability condition $x(t) \leq r^{o}$ for $t>0$.

\section{Proof:}

The reference signal $r^{o} \cdot 1\left(t-T_{f b}\right)$ models the fact that the space that is free at the bottleneck buffer, that is $\left(r^{o}-x(t)\right)$, reaches the source after the backward propagation delay $T_{f b}$ as $\left(r^{o} \cdot 1\left(t-T_{f b}\right)-x\left(t-T_{f b}\right)\right)$.

Using the controller (4), the input-output dynamics of the system in Fig. 5 is equal to the input-output dynamics of the system in Fig. 9. Thus, considering the system in Fig. 9, where $K(s)=k$, the Laplace transform of the output in response to the reference signal $r^{o} \cdot 1\left(t-T_{f b}\right)$ is

$$
X_{r}(s)=\frac{r^{\prime}}{s} \frac{1}{(1+s / k)} \cdot e^{-s T}
$$

By transforming $X_{r}(s)$ back to time domain, it follows:

$$
x_{r}(t)=r^{o} \cdot\left(1-e^{-k(t-T)}\right) \cdot 1(t-T)
$$


Moreover, using the controller (4), the transfer function from the available bandwidth $d(t)$ to the queue level $x_{d}(t)$ is given by (see Fig. 5)

$$
\frac{X_{d}(s)}{D(s)}=-\frac{1}{s}+\frac{k}{s(s+k)} e^{-T s}
$$

which, for $D(s)=\frac{a}{s}$, gives:

$$
x_{d}(t)=a\left(-t \cdot 1(t)+(t-T) \cdot 1(t-T)-\frac{1}{k}\left(1-e^{-k(t-T)}\right) \cdot 1(t-T)\right) \leq 0
$$

Thus, it follows that

$$
x(t)=x_{r}(t)+x_{d}(t) \leq x_{r}(t)=r^{o}\left(1-e^{-k(t-T)}\right) \cdot 1(t-T) \leq r^{o} \text { for } t>0 .
$$

This completes the proof.

Remark 3: The queue dynamics is characterised by the time constant $\tau=1 / k$. Therefore, the transient dynamics can be considered exhausted after the time $T_{t r}=T+4 \tau$.

\subsection{Full utilisation of network links and max-min fairness}

The efficiency condition must be satisfied to guarantee full utilisation of network links in the presence of time varying ABR bandwidth.

Proposition 2: The controller (4) guarantees the full utilisation of network links in the presence of the worst case disturbance $a \cdot 1(t)$ if the per$\mathrm{VC}$ capacity of network queues satisfies the following condition

$$
r^{o}>a(\tau+T)
$$

\section{Proof:}

Considering the queue dynamics in response to the step input $r^{0} \cdot 1\left(t-T_{f b}\right)$ and to the disturbance $a \cdot 1(t)$, it results 


$$
x(t)=x_{r}(t)+x_{d}(t)
$$

with

$$
\begin{aligned}
& x_{r}(t)=r^{o}\left(1-e^{-k(t-T)}\right) \cdot 1(t-T) \text { and } \\
& x_{d}(t)=a\left(-t \cdot 1(t)+(t-T) \cdot 1(t-T)-\frac{1}{k}\left(1-e^{-k(t-T)}\right) \cdot 1(t-T)\right) .
\end{aligned}
$$

For $t \gg T+4 \tau$, it results:

$$
x(t)=x_{s}=r^{o}-a \cdot T-\frac{a}{k}
$$

In order to fully utilise the bandwidth, $x_{s}$ must be greater than zero so that the queue has always data to send. Thus, condition (7) is derived.

Proposition 3: The buffer capacity $r^{o}=a \cdot(T+\tau)$ ensures that, in steady state condition, the ABR flow captures all available bandwidth $d(t)=a \cdot 1(t)$.

\section{Proof:}

Let $x_{s}$ and $u_{s}$ be the steady state values of $x(t)$ and $u(t)$. From Equation (5) it follows

$$
k\left(r^{o}-x_{s}-u_{s} T\right)=u_{s}
$$

which gives

$$
u_{s}=\frac{r^{o}-x_{s}}{\tau+T}
$$

Substituting (8) in (9) it results

$$
u_{s}=a
$$

that is, all ABR bandwidth is captured by the considered connection.

Proposition 4: The round-robin service of per-VC queues guarantees the fair allocation of available bandwidth to each VC. 


\section{Proof:}

The round-robin service discipline allocates the available bandwidth to each VC flow in accordance with a given definition of fairness such as, for instance, max-min fairness. In this way, the queuing service discipline, which is executed at the switch, allocates the fraction $a_{j}$ of the available bandwidth to the $j$-th VC. This bandwidth $a_{j}$ represents the disturbance for the $j$-th controlled VC. Finally, notice that the queuing service discipline is not "seen" by the congestion control algorithm, which is executed at the source, that is, the issues of congestion control and fairness are completely uncoupled.

\section{DISCRETE TIME CONTROL EQUATION}

By assuming a fluid model approximation of cell flows, a continuos time model of traffic and queue dynamics in ATM networks has been derived (Fig. 5). However, the feedback information is relayed in RM cells, and thus not available in continuos time, but rather in sampled form. Therefore the control equation (5) has to be transformed to discrete time form. This means that the controller updates the input rate every $T_{s}$ units of time, where $T_{s}$ is the sampling time. From Shannon sampling theorem and digital control theory (Astrom and Wittenmark, 1984), it is known that, in order to have a "continuous like" performance of the system under digitised control, the ratio of the time constant of the system over the sampling time must fall within the interval $(2,4)$, i.e.

$$
\tau / T_{s} \in(2,4)
$$

To write the discrete time version of the control equation (5), two cases are considered (Mascolo, Cavendish and Gerla, 1996):

1) $R T T \geq T_{s}$

It can be introduced the integer $m$ and the real $\varepsilon \in[0,1)$ so that $R T T / T_{s}=m+\varepsilon$. The discrete time control equation, at $t_{k}=k T_{s}$, gives the input rate

$$
u\left(t_{k}\right)=k\left[r^{o}-x\left(t_{k}-T_{f b}\right)-u\left(t_{k}-(m+1) T_{s}\right) \cdot \varepsilon T_{s}-\sum_{i=1}^{m} u\left(t_{k}-i T_{s}\right) \cdot T_{s}\right]
$$

2) $R T T<T_{s}$ 


$$
u\left(t_{k}\right)=k\left|r^{o}-x\left(t_{k}-T_{f b}\right)-u\left(t_{k}-T_{s}\right) \cdot R T T\right|
$$

Finally, we remark that the discrete time form of the control equation is simpler to be realised than the continuos one (Astrom and Wittenmark, 1984).

\section{SIMULATION RESULTS}

Computer simulations are carried out to investigate the dynamic behaviour of an ABR input rate sharing a bottleneck link with $(\mathrm{ABR}+\mathrm{VBR}+\mathrm{CBR})$ traffic. The available ABR bandwidth, normalised to unity, is modelled by means of an unknown and bounded disturbance function $d(t)$. A constant gain $k$ equal to $1 / 50$ and a buffer capacity $r^{\circ}$ equal to 50 are assumed in equation (5).

Fig. 10 shows the discrete time model of the system as it appears in the SIMULINK block diagram window. SIMULINK for MATLAB is a tool widely used by control engineers for simulating dynamic systems (Simulink, 1992). The use of this package is easy and allows programmers to save the effort to develop discrete event simulations.

Figs. 11-13 show the dynamics of $d(t), x(t)$ and $u(t)$. The ABR connection is characterised by the bandwidth-delay product of 20 cells that is typical of a LAN. Figs. 14-16 show the corresponding results for a connection characterised by a bandwidth-delay product of 80 cells, which is typical of a metro or regional WAN.

Simulation results confirm theoretical analysis. The ABR steady state input rate $u_{s}$ captures all available bandwidth, and the steady state queue value $x_{s}$ is in accordance with (8). 


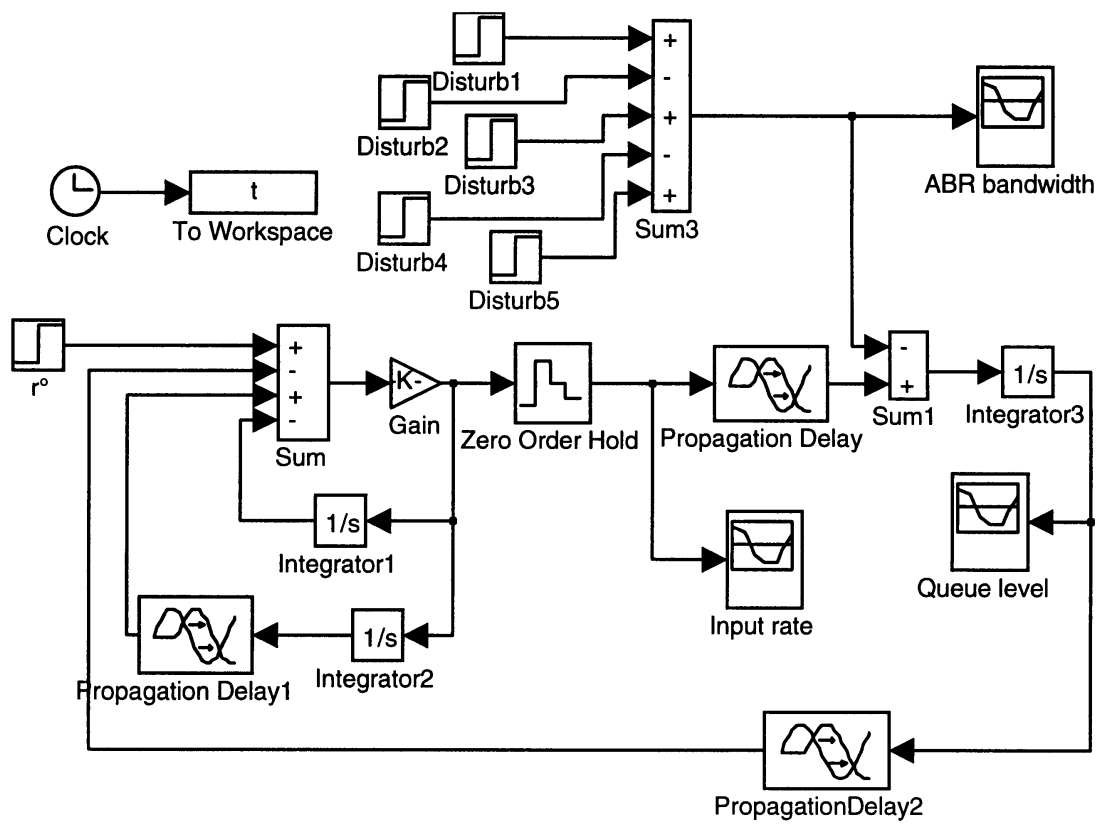

Fig. 10: Model of the controlled system as it appears in the SIMULINK block diagram window. 


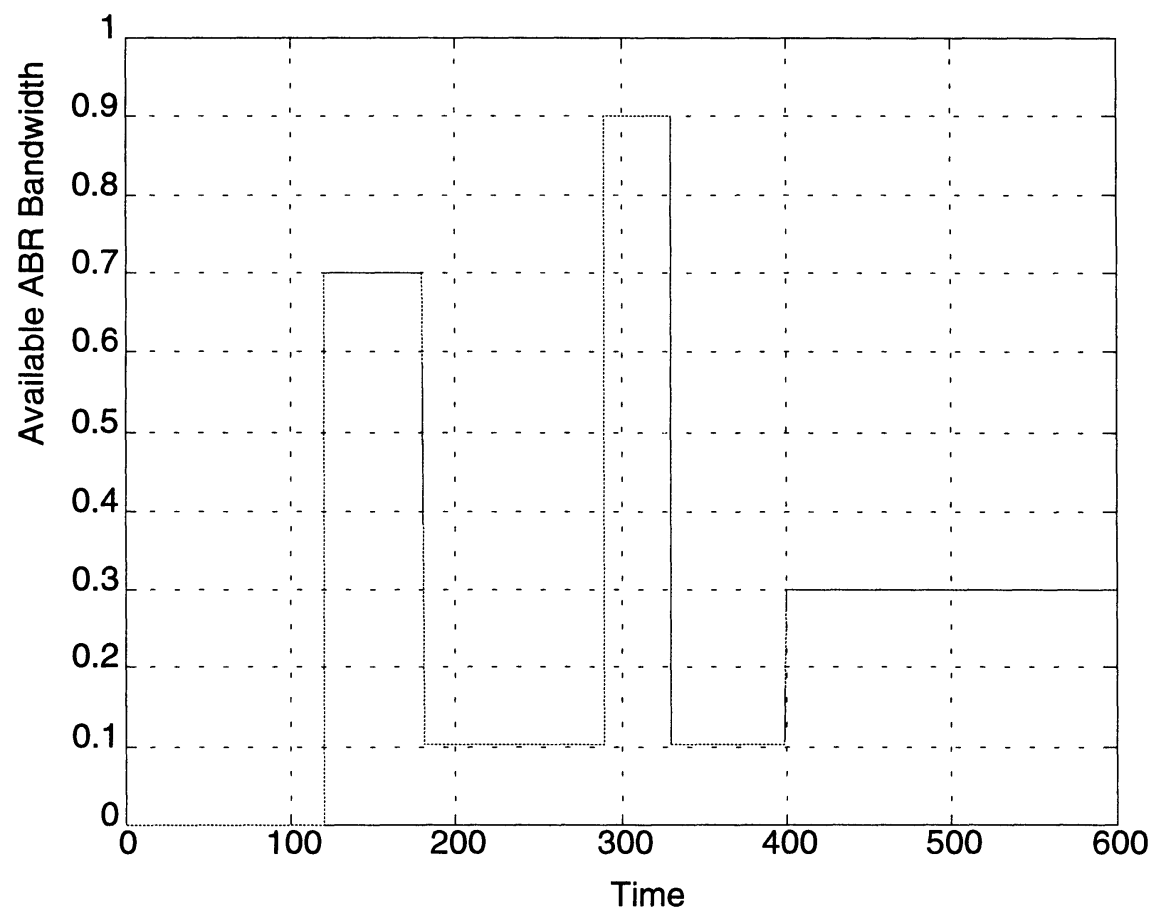

Fig. 11: Time varying ABR bandwidth $d(t)$ 


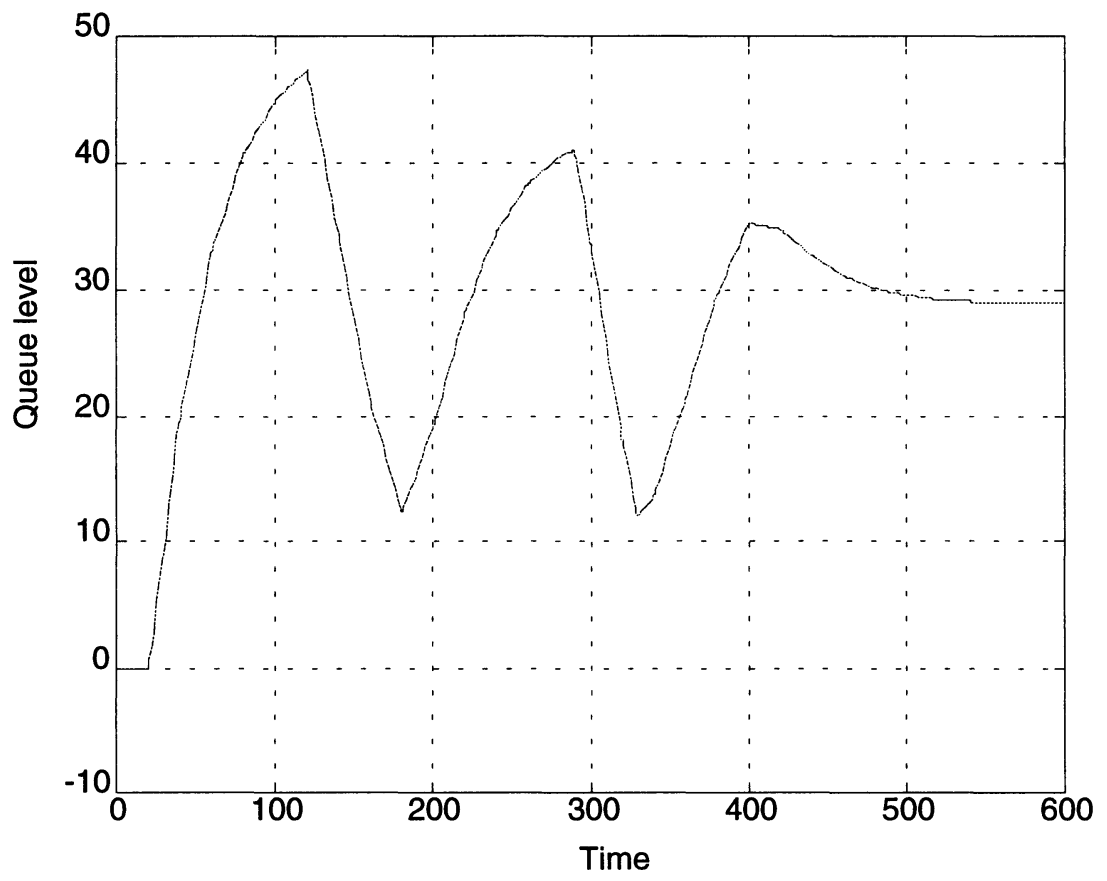

Fig. 12: Bottleneck queue level dynamics $x(t)$ 


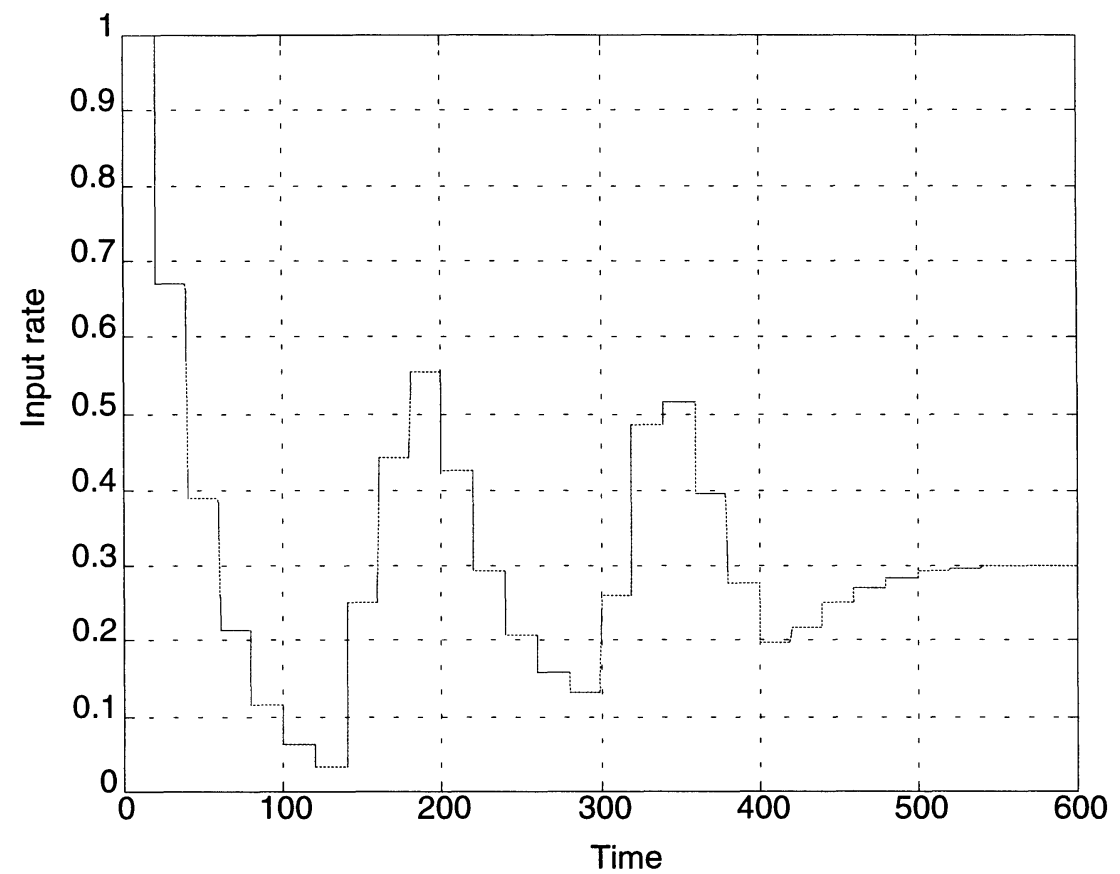

Fig. 13: Controlled ABR input rate $u(t)$ 


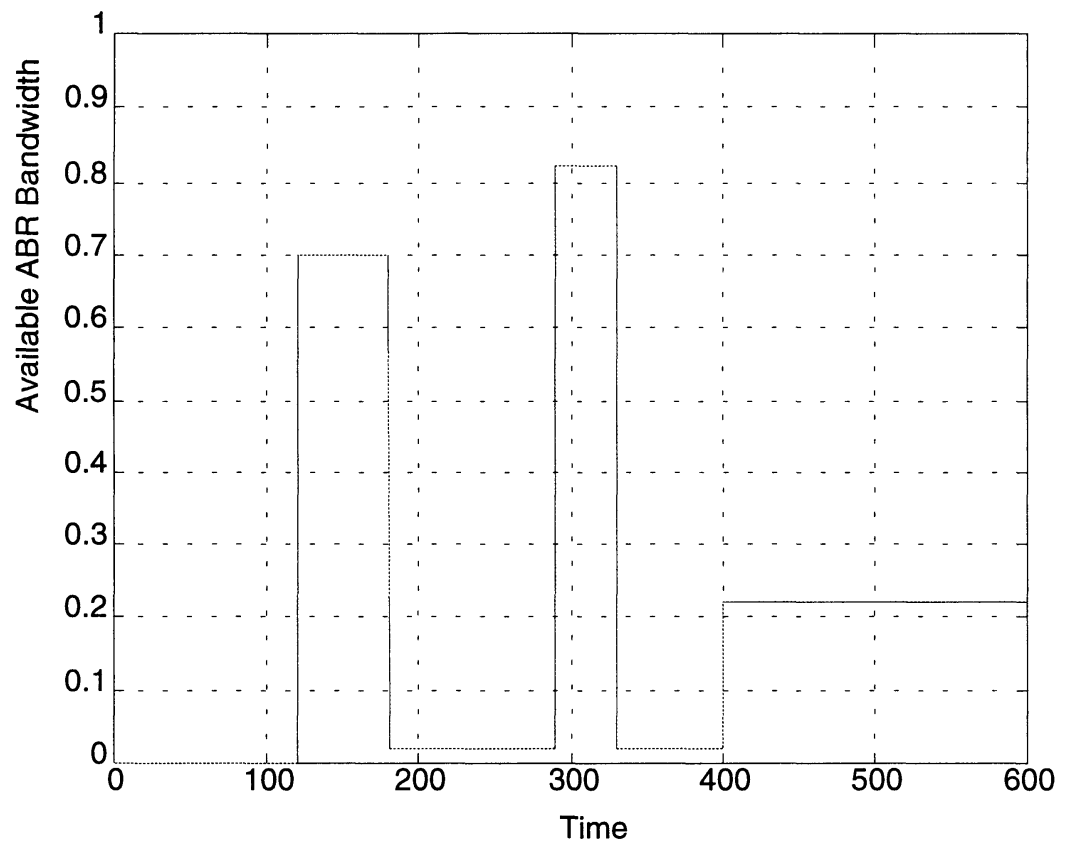

Fig. 14: Time varying ABR bandwidth $d(t)$ 


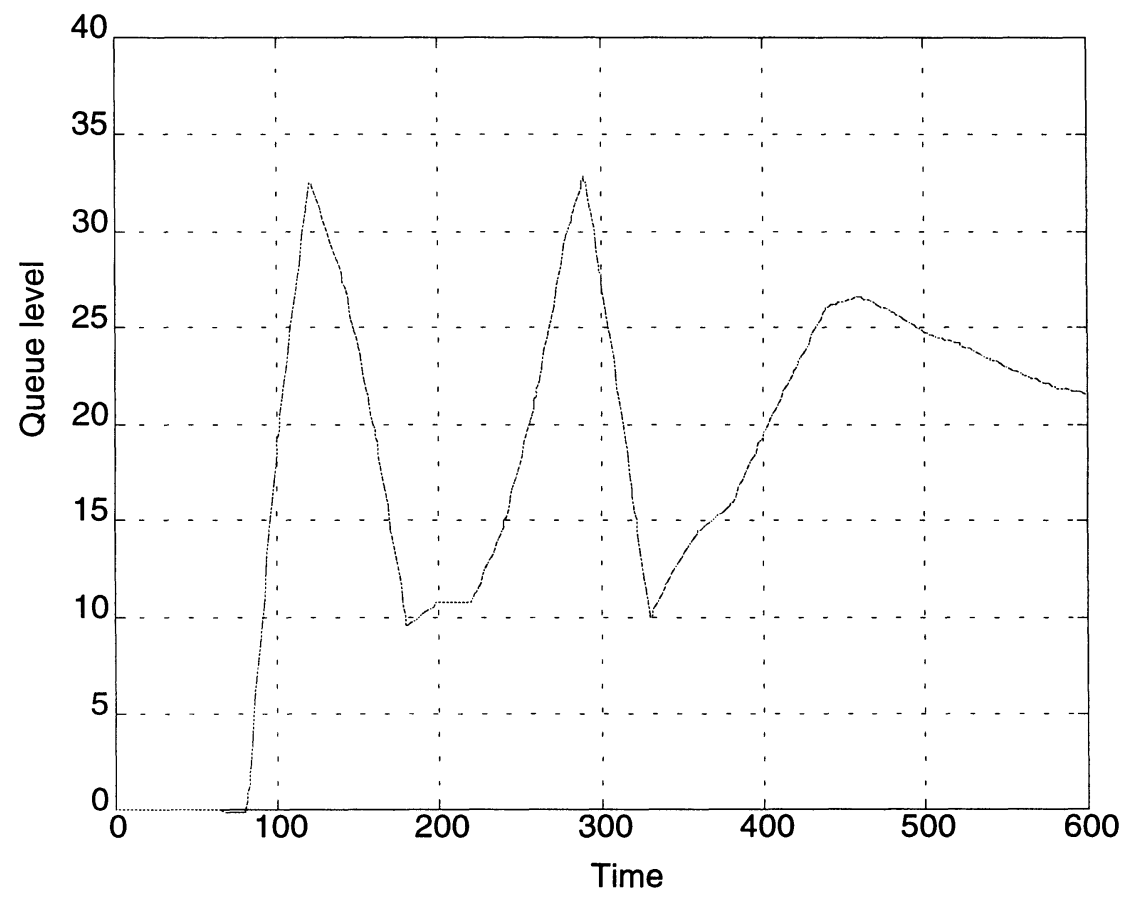

Fig. 15: Bottleneck queue level dynamics $x(t)$ 


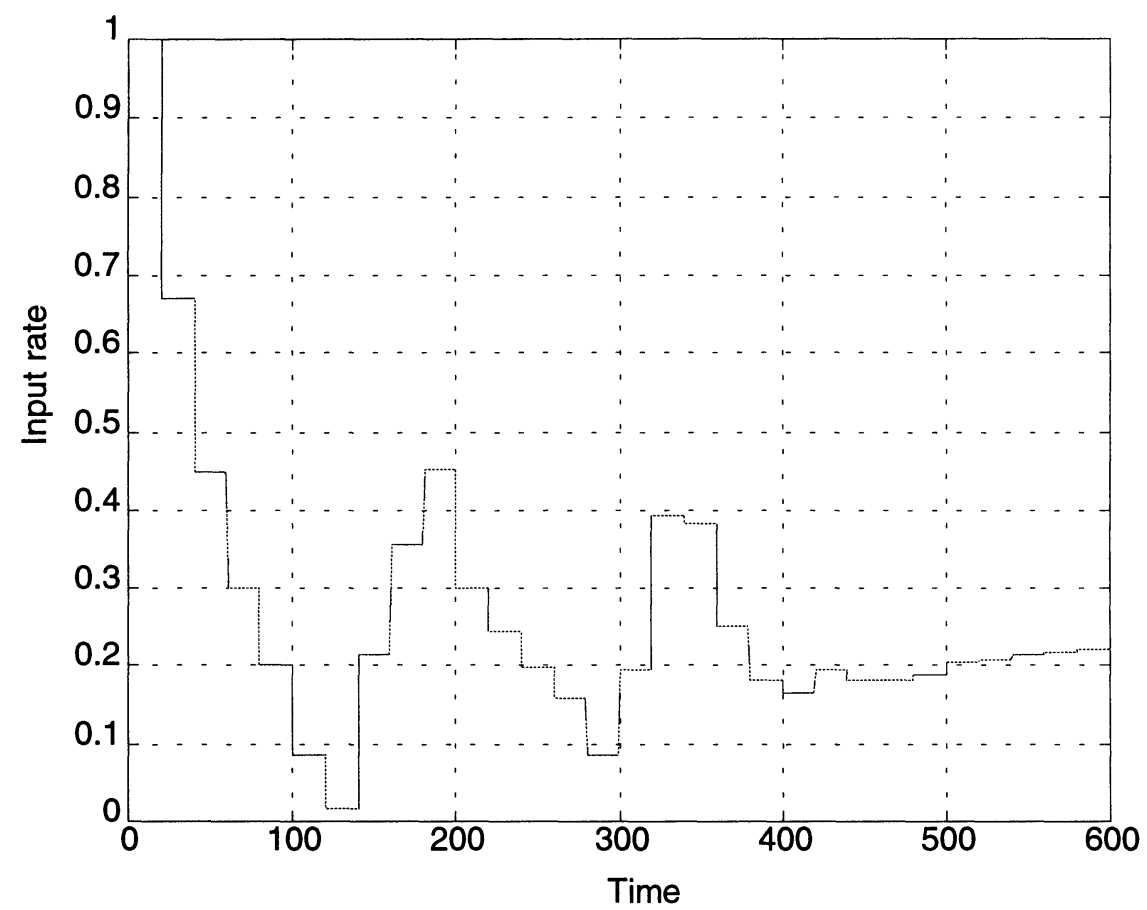

Fig. 16: Controlled ABR input rate $u(t)$

\section{CONCLUSION}

Classical linear control theory has been exploited to propose a general framework for modelling, designing and performance evaluation of congestion control algorithms for ATM networks. In particular, Smith's principle has been proposed as a convenient technique for controlling $A B R$ traffic in data networks characterised by a large bandwidth-delay product. The proposed algorithm has several advantages. In particular: 1) it is simple and effective in a realistic scenario where many connections, with different round trip times, share a bottleneck link that can move along the connection path; 2) it guarantees no cell loss and full utilisation of network links; 3) it considers the interaction of ABR with VBR traffic by means of an unknown and bounded disturbance function that represents the available bandwidth. This makes unnecessary for the switches to measure the available bandwidth, a difficult task in presence of a bursty traffic such as the VBR 
traffic; 4) the controlled system dynamics is a first order one, that is, the output exponentially converges to steady state value.

Performance evaluation has been also carried out by means of computer simulations using SIMULINK for MATLAB. The experimental results confirm the validity of the control theoretical model. Related and ongoing works are reported in (Cavendish, Gerla and Mascolo, 1995; Cavendish, Mascolo and Gerla, 1996; Mascolo and Gerla, 1997; Mascolo, Cavendish and Gerla, 1997; Mascolo, 1997).

\section{ACKNOWLEDGEMENTS}

I would like to thank my parents who generously supported the efforts for developing this work.

\section{REFERENCES}

Astrom K. J., Wittenmark B. (1984), Computer controlled systems, Prentice Hall, Englewood Cliffs, N. J.

ATM Forum Technical Committee TMWG (1996), ATM Forum Traffic Management Specification Version 4.0, ATM Forum/95-0013R11.

Benmohamed, L. and Meerkov S. M. (1993), Feedback Control of Congestion in Packet Switching Networks: The Case of a Single Congested Node, in IEEE/ACM Trans. on Networking, 1, (6), 693708.

Benmohamed, L. and Meerkov S. M. (1994), Feedback Control of Congestion in Packet Switching Networks: The Case of Multiple Congested Node, in Proc. of the American Control Conference, 11041108 .

Bonomi F., Mitra D., and Seery J. B. (1995), Adaptive Algorithms for Feedback-Based Flow Control in High-Speed, Wide-Area ATM Networks, in IEEE Journal on Selected Areas in Communications, 13, (7), 1267-1283.

Cavendish D., Gerla M., Mascolo S. (1995), ATM Rate Based Congestion Control Using a Smith Predictor: Implementation Issues, in First Workshop on ATM traffic Management, IFIP-WATM'95, Paris, 289296. 
Cavendish D., Mascolo S., Gerla M. (1996), Rate Based Congestion Control for multicast ABR traffic, in Proc. of Globecom 96, London, 11141118.

Charny A., Clark D. and Jain R. (1995), Congestion Control with Explicit Rate Indication, in Proc. of IEEE ICC'95, Seattle, 1954-1963.

Fendick K. W., Rodrigues M. A. and Weiss A. (1992), Analysis of a Ratebased Feedback Control Strategy for Long Haul Data Transport, in Performance Evaluation (16), 1992, 67-84.

Franklin G. F., Powell J. D., Emami-Naeini A. (1994), Feedback Control of Dynamic systems, Addison Wesley.

Fulton C., Li S. (1997), An ABR feedback control scheme with tracking, in Proc. of IEEE Infocom97, Kobe, Japan.

Iliadis I. (1995), A New Feedback Congestion Control Policy for Long Propagation Delays, in IEEE Journal on Selected Areas in Communications, 13, (7), 1284-1295.

Izmailov R. (1995), Adaptive Feedback Control Algorithms for Large Data Transfer in High-Speed Networks, in IEEE Trans. on Automatic Control, 40, (8), 1469-1471.

Jacobson V. (1988), Congestion Avoidance and Control," in Proc. of the SIGCOMM'88 Symposium, Stanford, CA, 314-329.

Jaffe J. (1981), Bottleneck Flow Control, in IEEE Trans. on Comm. 29, (7), 954-962.

Jain R. (1996), Congestion Control and Traffic Management in ATM Networks: Recent Advances and a Survey, Computer Networks and ISDN Systems, 28, (13), 1723-1738.

Kolarov A., Ramamurthy G. (1997), A Control Theoretic Approach to the Design of Closed Loop Rate Based Flow Control for High Speed ATM Networks, in Proc. of IEEE Infocom97, Kobe, Japan.

Marshall J. E. (1979), Control of time-delay systems, Peter Peregrinus Ltd. 
Mascolo S., Cavendish D., Gerla M. (1996) ATM Rate Based Congestion Control Using a Smith Predictor: an EPRCA Implementation, in Proc. IEEE Infocom96, S. Francisco, CA, 569-576.

Mascolo S., Cavendish D., Gerla M. (1997) ATM Rate Based Congestion Control Using a Smith Predictor, in Performance Evaluation, 31, (12), 51-65, Special Issue on ATM traffic Control.

Mascolo S. and Gerla M. (1997), Classical control approach to congestion control in high speed ATM networks, in Proc. of IEEE ATM 97 Workshop, Lisboa, Portugal, 361-367.

Mascolo S. (1997), Smith's principle for congestion control in high speed ATM networks, in Proc. of IEEE Conference on Decision and Control, San Diego, CA, 4595-4600.

Peterson, L. L. and Davie B. S. (1996), Computer Networks: a systems approach, Morgan Kaufmann Publishers, San Francisco, CA, 1996

Rohrs C. E., Berry R. A. (1997), A Linear Control Approach to Explicit Rate Feedback in ATM Networks, in Proc. of IEEE Infocom97, Kobe, Japan.

SIMULINK (1992), A Program for Simulating Dynamic Systems, The Math Works Inc.

Smith O.J. (1959), A Controller to Overcome Dead Time, ISA J., 6, (2), 2833.

Varaiya P. and Walrand J. (1996), High-performance Communication Networks, Morgan Kaufmann Publishers, San Francisco, CA, 1996.

Yin N., Hluchyj M. G.(1994), On Closed Loop Rate Control for ATM Cell Relay Networks, in Proc. Infocom94, 99-108.

Zhao Y., Li S. Q., and Sigarto S. (1997), A linear dynamic model for design of stable explicit-rate ABR control schemes, in Proc. of IEEE Infocom 97 , Kobe, Japan. 
PART SIX

\section{Resource Allocation}

